\title{
High-Fidelity Meshes from Tissue Samples for Diffusion MRI Simulations
}

\author{
Eleftheria Panagiotaki ${ }^{1}$, Matt G. Hall ${ }^{1}$, Hui Zhang ${ }^{1}$, Bernard Siow ${ }^{1,2}$, \\ Mark F. Lythgoe ${ }^{2}$, and Daniel C. Alexander ${ }^{1}$, \\ 1 Centre for Medical Image Computing, Department of Computer Science, \\ University College London, UK \\ E.Panagiotaki@cs.ucl.ac.uk \\ 2 Centre for Advanced Biomedical Imaging, University College London, UK
}

\begin{abstract}
This paper presents a method for constructing detailed geometric models of tissue microstructure for synthesizing realistic diffusion MRI data. We construct three-dimensional mesh models from confocal microscopy image stacks using the marching cubes algorithm. Randomwalk simulations within the resulting meshes provide synthetic diffusion MRI measurements. Experiments optimise simulation parameters and complexity of the meshes to achieve accuracy and reproducibility while minimizing computation time. Finally we assess the quality of the synthesized data from the mesh models by comparison with scanner data as well as synthetic data from simple geometric models and simplified meshes that vary only in two dimensions. The results support the extra complexity of the three-dimensional mesh compared to simpler models although sensitivity to the mesh resolution is quite robust.
\end{abstract}

\section{Introduction}

Diffusion-Weighted Magnetic Resonance Imaging (DW-MRI) is a non-invasive imaging technique sensitive to the dispersion of water molecules in biological tissues. It has become an essential tool for probing microstructure. Diffusion Tensor Imaging (DTI) indices such as mean diffusivity and diffusion anisotropy provide useful but non-specific markers of white matter integrity. More recent techniques 1 12 2 34 aim to estimate specific features of tissue microstructure such as axon diameter and density. However, questions remain about how well these microstructural indices from diffusion MRI reflect the actual tissue microstructure since it is difficult to obtain ground truth information.

Synthetic data is a powerful tool for developing, optimizing, evaluating and comparing diffusion MRI techniques. Unlike scanner data, it provides a ground truth, thereby allowing performance evaluation of methods in a controlled environment. One approach for generating synthetic data is to use a simple model like the DT [5] which describes the displacement of particles with a Gaussian distribution. This simple model provides measurements with negligible computation cost. However, due to its simplicity the DT model ignores features of the tissue and the diffusion process within. In particular, it does not account

T. Jiang et al. (Eds.): MICCAI 2010, Part II, LNCS 6362, pp. 404411, 2010.

(C) Springer-Verlag Berlin Heidelberg 2010 
for restricted diffusion within cells so oversimplifies the signal from real tissue. To address this limitation, recent diffusion MRI studies [123467] use a variety of multi-compartment models that separate restricted, hindered and free diffusion. Analytic compartmental models are computationally efficient but are approximations and limited to describing diffusion in simple geometries such as cylinders and spheres.

This has motivated work on numerical methods, which allow arbitrary complexity in the diffusion environment and measurement process. The most common numerical methods for synthesizing diffusion MRI data are based on finitedifference approaches e.g. 8] and Monte-Carlo methods e.g. 91011]. Finitedifference methods rely on an approximate solution to the diffusion equation at a discrete number of points, e.g. on a grid, while Monte-Carlo methods simulate Brownian motion of spins within geometric models of tissue. Numerical methods can investigate diffusion environments that are impossible using analytic models. For example, Hall and Alexander 10, use Monte-Carlo simulations to synthesize data in a model of tissue undergoing swelling to simulate oedema where cylindrical axons swell and abut.

Other work 811] base the simulations in models derived from light microscopy images for a better approximation of the tissue. Lipinski [1] was the first to use histologic images in combination with two-dimensional Monte-Carlo simulations to study the diffusion signal. The tissue model is based on a rough segmentation of a digitized light microscopy image of white matter tissue. More recently, Chin et al. 8] use a finite-difference approach and construct a tissue model by replicating a light microscopy image in the third dimension. These numerical models are an improvement on the simplified geometric models, yet, the singleslice microscopy images are low-resolution and the models describe the tissue only in two dimensions. However, numerical methods are more computationally intensive than analytic models and the implementation of complex realistic environments is non-trivial, causing optimization and tuning of the simulation to be especially challenging.

This paper presents a method for constructing three-dimensional tissue models from a stack of high-resolution Confocal Laser Scanning Microscopy (CLSM) images. We capture the three-dimensional structure of biological tissue in a more natural way than previous studies 811. We demonstrate the method using a biological phantom (asparagus) which is a useful model with similar microstructure to white matter [12]. Experiments vary simulation parameters and mesh properties to optimize the precision of the synthesized data while minimizing computational complexity. We compare results to scanner data and to synthetic data from simple parametric models and two-dimensional meshes.

Section 2 describes the method for constructing and evaluating three-dimensional tissue models. Section 3 outlines the experiments for optimizing simulation parameters, exploring the effect of mesh resolution, and evaluating synthetic data from the mesh by comparison with scanner data and with data generated from simpler models. Section 4 concludes. 


\section{Methods}

The full procedure has several stages. First, we acquire DW-MRI data with a wide range of diffusion times and diffusion weightings with gradient directions both parallel and perpendicular to the asparagus stem. We identify a region of interest (ROI) in the DW-MRI data containing one of the vascular bundles (Fig 1a), which we cut from the stem and image with CLSM (Fig 1b) to obtain a stack of images. The vascular bundles in the asparagus consist of highlyorganised cylindrical fibres with thick walls that exhibit anisotropic diffusion and the distribution of capillary sizes is similar to brain white matter tissue 12. We construct the three-dimensional mesh model (Fig 1k) with the marching cubes algorithm [13 and use it as a substrate in Monte-Carlo simulations [10] to synthesize DW-MRI data.
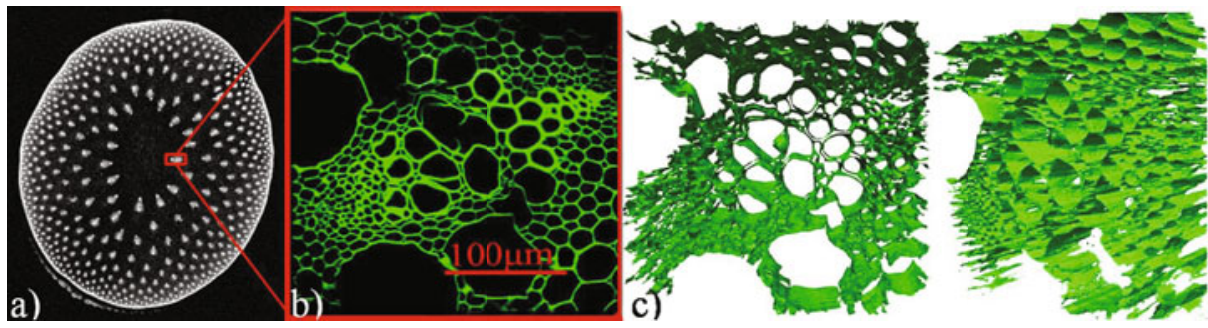

Fig. 1. a) DW-MRI image of a transverse section of the asparagus stem. The red square indicates the ROI, containing one of the vascular bundles, appearing white in the MRI image. b) CLSM image of the same ROI. c) three-dimensional mesh model shown from two different angles.

Sample preparation: We place a stem of green asparagus (Asparagus officinalis) in a syringe padded with cotton soaked in pure water. This keeps the sample hydrated, preventing shrinkage and diffusion changes during the scan.

MRI Acquisition: We acquire DW-MR images with a small bore 9.4T scanner (Varian) with maximum gradient strength $400 \mathrm{mT} / \mathrm{m}$ and use a controlled air-flow mechanism to keep the sample temperature constant within $\pm 1^{\circ} \mathrm{C}$. The two-direction encoding scheme has one direction parallel to the asparagus stem and one perpendicular. We acquire 64 pulsed-gradient spin-echo (PGSE) measurements with six diffusion times, $\Delta=10,30,50,70,80,100 \mathrm{~ms}$, three gradient durations $\delta=3,10,20 \mathrm{~ms}$ and gradient strength $|\mathbf{G}|$ varied from 40 to $400 \mathrm{mT} / \mathrm{m}$ in ten steps of $40 \mathrm{mT} / \mathrm{m}$. The 64 measurements includes all combinations with $\Delta>\delta$ and $b<6.5 \times 10^{9} \mathrm{sm}^{-2}$. We use the minimum echo time (TE) possible for each measurement and set the repetition time (TR) to $3 \mathrm{~s}$. The total acquisition time is approximately 40 hours. We correct for $T_{2}$ dependence by acquiring separate $b=0$ images for each combination of $\delta$ and $\Delta$. The in-plane field of view is $16 \mathrm{~mm}$. The matrix size is $256 \times 256$ and the slice thickness is $0.5 \mathrm{~mm}$. 
Confocal Acquisition: We cut out four $600 \mu \mathrm{m}$ thick sections which we then stain with Eosin for 10 minutes and wash thoroughly with phosphate buffered saline. To avoid any geometric distortions we use vibratome, which is designed for sectioning soft tissue without freezing or paraffin, and choose to image only the middle slices. We use a Leica SP2 AOBS confocal multi-photon laser scanning microscope coupled to a Leica DMRE upright microscope (Leica, Milton Keynes, UK). We receive the laser output with an electro-optical modulator (EOM) (Linos LIV20) before delivering to the confocal microscope through a series of optical mirrors. The EOM allows the laser intensity at the objective to be controlled and optimized. The EOM is set at $90 \%$ for imaging to ensure that the polarization of the incidental laser beam remained consistent across all specimens. We image the specimens with a $40 \times 1.25 \mathrm{NA}$ oil Plan Apo objective to give image dimensions of $315 \mu \mathrm{m} \times 315 \mu \mathrm{m}$. We acquire optical z-sections of $1 \mu \mathrm{m}$ thickness reaching a maximum depth of $100 \mu \mathrm{m}$ with an image averaging set to 3 per z-slice. The image size is $1024 \times 1024$ pixels.

Model Construction: To construct the mesh models we assemble the images into a stack and segment them by thresholding to create binary images that separate the intra- and extra-capillary space. The intra-cellular volume fraction is determined by counting the pixels below the threshold. We use the marching cubes algorithm [13] on the binary stack to produce the three-dimensional mesh model. Memory limitations require that we downsample the 100 CLSM images to $144 \times 144$ pixels while keeping the three-dimensional aspect ratio of the voxels the same as the original image stack, which also makes computation times manageable. The algorithm produces a mesh of around 500,000 triangles.

Simulations: The simulation system in [10] generates synthetic measurements for each combination of scan parameters from diffusing spins constrained by the structure of the mesh. Each triangle in the mesh acts as an impermeable reflecting boundary. For computational efficiency the system checks only for intersections with triangles within the range of each step. Spins are initialized uniformly across the mesh, in both intra- and extra-cellular regions. Here we assume the same properties (i.e. diffusivity, relaxation times) for both regions.

\section{Experiments}

This section outlines three sets of experiments. The first identifies a suitable combination of number of spins and updates within a fixed simulation runtime. The second explores the effect of the mesh resolution. The final experiment compares synthetic data from the three-dimensional mesh with scanner data and synthetic data from simpler geometric models.

\subsection{Simulation Parameter Optimization}

The aim is to maximize precision and accuracy of the synthetic measurements while minimizing computational cost. The complexity of the simulation is order $U=N T$ where $N$ is the number of spins and $T$ is the number of updates. 
Without a ground truth, the accuracy of the simulation is hard to establish. However, 10 shows on simpler substrates that with fixed $U$ accuracy tends to increase up to a certain $N$ and suddenly depart although standard deviation decreases. Here we search for the same trend to identify the best trade off.

We use the $144 \times 144$ mesh as substrate with the same acquisition protocol as the scanner data and diffusivity $d=2.1 \times 10^{-9} \mathrm{~m}^{2} / \mathrm{s}$, estimated from scanner measurements with low $b$ value using the monoexponential model. Simulations with various combinations of $N, T$ for $U=10^{8}$ are repeated 10 times with different initial spin positions. Each simulation runs in approximately 48 hours.

Results: The mean signal for the perpendicular direction over all scan parameter combinations and repeats remains similar as $N$ increases from low $N \simeq 1 \times 10^{4}$ until $N \simeq 1 \times 10^{5}$ for $b=1.5 \times 10^{9} \mathrm{sm}^{-2}$. All the measurements with different $b$ values have similar trends with small variations that do not affect the final choice.

Conclusions: As in [10], we observe a gradual increase in reproducibility as $N$ increases. The mean signal remains consistent from low $N$ until $N \simeq 9 \times 10^{4}$ above which it increases noticeably. The increase is most likely a bias introduced by the timesteps being too long. We choose $N=8 \times 10^{4}$ and $T=1250$ to keep reproducibility high while remaining safely within the region of short enough timesteps.

\subsection{Signal Dependency on Mesh Fidelity}

This experiment compares meshes constructed from different resolution CLSM images to investigate the effect of varying mesh resolution on synthetic DW-MRI data. The highest image resolution we use is $144 \times 144$ pixels and the lowest $9 \times 9$ pixels with four intermediate stages. We synthesize data from the different resolution meshes using the parameters from experiment 3.1. We calculate the Mean Squared Error $\left(M S E_{144}\right)$ compared to the $144 \times 144$ mesh to show differences in synthesizing data with different resolution meshes, and the $M S E_{M R I}$ compared to the scanner data to reveal which of these differences are significant.
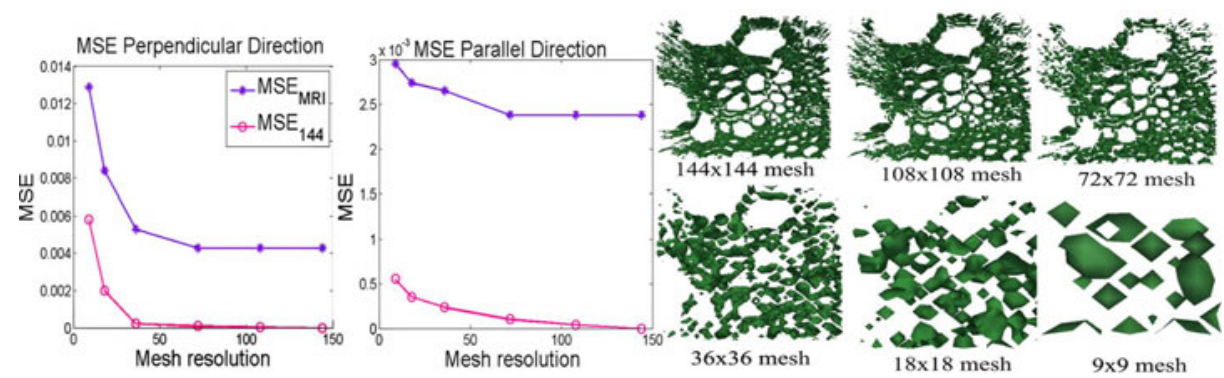

Fig. 2. Left: Plots of the MSE of the signal in the parallel and perpendicular direction in comparison with the high-resolution mesh and the scanner data against mesh resolution. Right: Illustration of the different complexity meshes used in the simulations. 
Results: Figure 2 plots the $M S E_{144}$ and $M S E_{M R I}$ of the normalised signal for both directions against mesh resolution. We exclude all measurements with $S<$ 0.1 from the plots and the MSE calculations e.g. to avoid significant noise-floor effects. In the perpendicular direction the $M S E_{144}$ shows very little variation between the $144 \times 144$ and $36 \times 36$ resolution meshes. In the parallel direction, the $M S E_{144}$ starts to increase at $72 \times 72$ resolution. The $M S E_{M R I}$ however, shows little difference in both directions for the meshes with resolution $144 \times 144$ to $72 \times 72$.

Conclusions: In both directions the $M S E_{M R I}$ remains similar for the meshes with resolution $144 \times 144$ to $72 \times 72$ meshes. At $36 \times 36$ we see slight elevation that becomes more obvious in lower resolution meshes. The results suggest that $72 \times 72$ resolution approximates the geometry closely enough to capture variations in water diffusion that MR signals are sensitive to.

\subsection{Model Comparison with Scanner Data}

The last experiment assesses the quality of synthetic MRI data of the mesh models. For comparison, we generate three sets of synthetic data: the $72 \times 72$ three-dimensional from experiment 3.2 , an extruded two-dimensional mesh models and a packed-cylinder substrate with constant radius. To construct the extruded mesh we choose an image from the stack we used for the three-dimensional mesh model with $f=0.8$ which is consistent with the three-dimensional model, replicate it to have the same number of slices used to generate the $72 \times 72$ mesh and run the same meshing algorithm. The packed-cylinder substrate has squarepacked cylinders with radius $25 \mu \mathrm{m}$. The choice of single radius comes from the mean capillary radius in the mesh weighted by capillary volume [4]. We pick the packing density so that the intra-capillary volume fraction is also $f=0.8$.
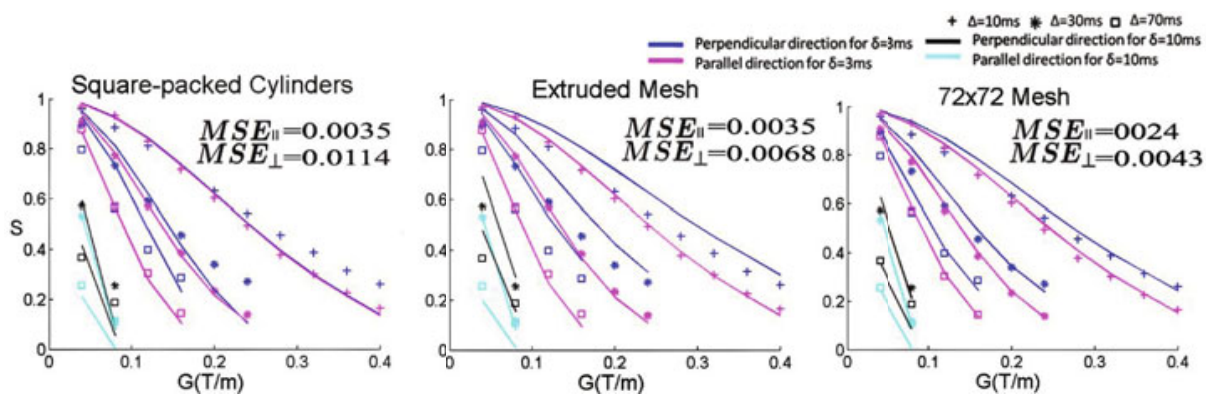

Fig. 3. Data synthesized from the cylinder model, the extruded mesh and the threedimensional mesh with the scanner data from the PGSE experiment. For clarity, the normalised signal $S$ is plotted only for selected values of $\Delta$ and $\delta$ as a function of the gradient strength $|\mathbf{G}|$ for the parallel and the perpendicular direction. Markers show the scanner data; lines show the synthetic data. Mean-squared error, for the parallel and perpendicular direction, are included for each model. 
Results: Figure 3 compares data synthesized from the three models to the scanner data by plotting the normalised signal $S$ only for selected values of $\Delta$ and $\delta$ as a function of the gradient strength $|\mathbf{G}|$ for the parallel and perpendicular direction. Again we exclude all measurements with $S<0.1$ from the plots and the MSE calculations to avoid significant noise-floor effects. Predictions from simulations with the cylinders of constant radii and the extruded mesh model are unable to capture the data as well as the mesh model. For example the cylinders underestimate the perpendicular signals with $\Delta=10,30 \mathrm{~ms}$ and $\delta=$ 3, $10 \mathrm{~ms}$ while the extruded model overestimates them. The three-dimensional mesh model agrees closely in both directions for $\Delta=10,30,70 \mathrm{~ms}$ and $\delta=3,10$ ms. The MSE is lower for the three-dimensional mesh model in both directions.

Conclusions: The complex three-dimensional model minimizes the MSE and captures the data in both directions for $\Delta=10,30,70 \mathrm{~ms}$ and $\delta=3,10 \mathrm{~ms}$.

\section{Conclusions}

This work introduces a method for constructing a detailed tissue mesh model using CLSM to generate realistic diffusion MRI data. We investigate optimal simulation and mesh properties for precision and accuracy of the synthesized data. We test the simulated data from three-dimensional mesh models against scanner data, simpler extruded mesh models and simple parametric models. Results with the three-dimensional mesh model are very promising, agree with scanner data well and match the data better than the extruded and parametric models in both directions.

The method we propose in this paper can be refined in a number of ways. So far, we optimize the simulation parameters without a ground truth. In a similar experiment in [10] they compare results to an analytic model of restriction, however the mesh model here is much more complex and analytic solutions cannot provide a ground truth. Another aspect we could further explore is the choice of diffusivity. We could refine agreement by searching for the diffusivity that minimizes error between the synthetic data and the scanner data. The quality of the generated mesh also depends on the segmentation process and the downsampling of the confocal images. So far we use a simple thresholding algorithm. We could improve the accuracy and smoothness of the mesh by using more sophisticated segmentation algorithms. To avoid downsampling while preserving the topology with the minimum number of triangles we could use a meshing algorithm combined with decimation 14. However, preliminary experiments with decimation reveal problems with intersecting triangles that cause the simulation to fail. Exploitation of more sophisticated meshing remains a focus for further work.

The Monte-Carlo simulation approach with a tissue model of high fidelity provides a mechanism for high quality data synthesis for testing and developing diffusion MR algorithms. It also allows for testing of the influence of subtle effects such as permeability and surface-particle interactions (e.g. particles temporarily trapped on the surface) that analytic models cannot capture. Future work will 
extend the experiments to other samples in particular to brain tissue to allow testing and comparison of analytic models in a similar way to [7.

Acknowledgements. Thanks to Dr Chris Thrasivoulou for help with the confocal acquisition. Funded by EPSRC grants EP/E056938/1, EP/E007748, British Heart Foundation and BBSRC.

\section{References}

1. Stanisz, G.J., Szafer, A., Wright, G.A., Henkelman, R.M.: An analytical model of restricted diffusion in bovine optic nerve. Magnetic Resonance in Medicine 37, 103-111 (1997)

2. Assaf, Y., Basser, P.: Composite hindered and restricted model of diffusion (CHARMED) MR imaging of the human brain. NeuroImage 27, 48-58 (2005)

3. Alexander, D.C.: A general framework for experiment design in diffusion MRI and its application in measuring direct tissue-microstructure features. Magnetic Resonance in Medicine 60, 439-448 (2008)

4. Alexander, D.C., Hubbard, P.L., Hall, M.G., Moore, E.A., Ptito, M., Parker, G.J., Dyrby, T.B.: Orientationally invariant indices of axon diameter and density from diffusion MRI. NeuroImage (2010), doi:10.1016/j.neuroimage.2010.05.043

5. Basser, P.J., Mattiello, J., LeBihan, D.: MR diffusion tensor spectroscopy and imaging. Biophysical Journal 66, 259-267 (1994)

6. Behrens, T.E.J., Woolrich, M.W., Jenkinson, M., Johansen, H.: Characterization and propagation of uncertainty in diffusion-weighted MR imaging. Magnetic Resonance in Medicine 50, 1077-1088 (2003)

7. Panagiotaki, E., Fonteijn, H., Siow, B., Hall, M.G., Price, A., Lythgoe, M.F., Alexander, D.C.: Two-Compartment Models of the Diffusion MR Signal in Brain White Matter. In: Yang, G.-Z., Hawkes, D., Rueckert, D., Noble, A., Taylor, C. (eds.) MICCAI 2009. LNCS, vol. 5761, pp. 329-336. Springer, Heidelberg (2009)

8. Chin, C.L., Wehrli, F.W., Hwang, S.N., Takahashi, M., Hackney, D.B.: Biexponential diffusion attenuation in the rat spinal cord: Computer simulations based on anatomic images of axonal architecture. Magnetic Resonance in Medicine 47, 455-460 (2002)

9. Szafer, A., Zhong, J., Gore, J.C.: Theoretical model for water diffusion in tissues. Magnetic Resonance in Medicine 33, 697-712 (1995)

10. Hall, M.G., Alexander, D.C.: Convergence and Parameter Choice for Monte-Carlo Simulations for Diffusion MRI. IEEE Transactions on Medical Imaging 28, 1354 1364 (2009)

11. Lipinski, H.G.: Monte Carlo simulation of extracellular diffusion in brain tissues. Phys. Med. Biol. 35, 441-447 (1990)

12. Lätt, J., Nilsson, M., Rydhög, A., Wirestam, R., Ståhlberg, F., Brockstedt, S.: Effects of restricted diffusion in a biological phantom: a q-space diffusion MRI study of asparagus stems at a 3T clinical scanner. Magnetic Resonance Materials in Physics, Biology and Medicine 20(4), 213-222 (2007)

13. Lorensen, W.E., Cline, H.E.: Marching cubes: A high resolution 3D surface construction algorithm. Computer Graphics 21(4) (1987)

14. Schroeder, W., Zarge, J., Lorensen, W., et al.: Decimation of triangle meshes. CG NY ACM 26, 65 (1992) 